\title{
Molecular detection of Leishmania DNA and identification of blood meals in wild caught phlebotomine sand flies (Diptera: Psychodidae) from southern Portugal
}

\author{
Carla Maia ${ }^{1 *}$, Ricardo Parreira ${ }^{1,2}$, José Manuel Cristóvão ${ }^{1}$, Ferdinando Bernardino Freitas ${ }^{1}$, Maria Odete Afonso ${ }^{1}$
} and Lenea Campino ${ }^{1,3}$

\begin{abstract}
Background: Zoonotic visceral leishmaniasis caused by Leishmania infantum which is transmitted by phlebotomine sand flies (Diptera, Psychodidae) is endemic in the Mediterranean basin. The main objectives of this study were to (i) detect Leishmania DNA and (ii) identify blood meal sources in wild caught female sand flies in the zoonotic leishmaniasis region of Algarve, Portugal/Southwestern Europe.

Methods: Phlebotomine sand flies were collected using CDC miniature light traps and sticky papers. Sand flies were identified morphologically and tested for Leishmania sp. by PCR using ITS-1 as the target sequence. The source of blood meal of the engorged females was determined using the cyt-b sequence.

Results: Out of the 4,971 (2,584 males and 2,387 females) collected sand flies, Leishmania DNA was detected by PCR in three females $(0.13 \%)$, specifically in two specimens identified on the basis of morphological features as Sergentomyia minuta and one as Phlebotomus perniciosus. Haematic preferences, as defined by the analysis of cyt- $b$ DNA amplified from the blood-meals detected in the engorged female specimens, showed that $P$. perniciosus fed on a wide range of domestic animals while human and lizard DNA was detected in engorged S. minuta.
\end{abstract}

Conclusions: The anthropophilic behavior of $S$. minuta together with the detection of Leishmania DNA highlights the need to determine the role played by this species in the transmission of Leishmania parasites to humans. In addition, on-going surveillance on Leishmania vectors is crucial as the increased migration and travelling flow elevate the risk of introduction and spread of infections by Leishmania species which are non-endemic.

Keywords: Blood-meal, Leishmania, Phlebotomine sand flies, Phlebotomus perniciosus, Sergentomyia minuta, Portugal, Southwestern Europe

\section{Background}

Leishmaniasis caused by Leishmania infantum is the only tropical vector-borne disease that has been endemic in southern Europe for decades [1]. Most of the reported cases are due to zoonotic visceral leishmaniasis (VL), the most dangerous form of Leishmania infection, being lethal when untreated. Dogs are considered the major

\footnotetext{
* Correspondence: carlamaia@ihmt.unl.pt

'Unidade de Parasitologia Médica, Global Health and Tropical Medicine (GHTM), Instituto de Higiene e Medicina Tropical (IHMT), Universidade Nova de Lisboa (UNL), Lisbon, Portugal

Full list of author information is available at the end of the article
}

host for these parasites, and the main reservoir for human infections. In nature, the pathogen transmission occurs via the infective bite of phlebotomine sand flies (Diptera, Psychodidae), for both humans and dogs.

In Portugal, as in other countries in the south of Europe, VL was initially described as a pediatric disease but from the end of the 1980s onwards, the number of cases in children has decreased with a concomitant increase of infection in adults, commonly associated with HIV/AIDS [2]. In the last ten years (2005-2014), 119 new cases of human VL (17 in immunocompetent adults, 36 in children and 66 in immunocompromised patients) and 16 cutaneous

\section{Ciomed Central}

(C) 2015 Maia et al.; licensee BioMed Central. This is an Open Access article distributed under the terms of the Creative Commons Attribution License (http://creativecommons.org/licenses/by/4.0), which permits unrestricted use, distribution, and reproduction in any medium, provided the original work is properly credited. The Creative Commons Public Domain Dedication waiver (http://creativecommons.org/publicdomain/zero/1.0/) applies to the data made available in this article unless otherwise stated. 
leishmaniasis cases were diagnosed at the Leishmaniasis Laboratory at the Institute of Hygiene and Tropical Medicine. Leishmania infantum zymodeme MON-1 is the most common aetiological agent of autochthonous human and canine leishmaniasis cases [3] and Phlebotomus perniciosus and Phlebotomus ariasi have been confirmed as proven vectors [4].

As data regarding Leishmania infection rate and blood meal sources of phlebotomine sand flies in Portugal is still too scarce, this study was implemented so as to allow the (i) detection of Leishmania DNA and (ii) identification of blood meal sources in wild caught female sand flies in Algarve, Portugal/Southwestern Europe.

\section{Methods}

\section{Study area}

Algarve, located in southern Portugal, has an area of $5,412 \mathrm{Km} 2$ with an estimated number of permanent inhabitants approximating 450,000 [5], which triplicates during summer months. Figs (Ficus carica), almonds (Prunus amygdalus), oranges (Citrus sinensis), carobs (Ceratonia siliqua), strawberries trees (Arbutus unedo) and cork oaks (Quercus suber), are the most common crops in the region [6]. Algarve has a Mediterranean climate with warm weather (annual average temperature of $18^{\circ} \mathrm{C}$ ) and low rainfall almost all year round (annual average of $500 \mathrm{~mm}$ ). Summer (June-September) is the driest and warmest season with average monthly temperatures between $16^{\circ}$ and $28-30^{\circ} \mathrm{C}$ (www.ipma.pt).

\section{Collection and identification of sand flies}

Between May to October from 2011 to 2013, CDC light traps and sticky oil papers were set up in 11 sampling points during three consecutive days per month. Collection places included domestic, peri-domestic and sylvatic environments. In most of the studied biotopes, in addition to humans and dogs, the major vertebrates visible within a $50 \mathrm{~m}$ radius of the collection spots were livestock, horses, pigs, rabbits and poultry. Collected sand flies were stored in $70 \%$ ethanol for further analysis. A total of 4,971 sand flies (2,584 males and 2,387 females) were collected and identified morphologically. Phlebotomine specimens of both genders were identified by their morphological characteristics to the species level, according to Pires [7]. Female identification was done by microscopic observation of the spermatheca, after dissection and mounting of the three last abdominal segments in Marc-André solution, while males were identified by direct stereomicroscopic observation of the genitalia. In addition, for each female, the presence of eggs (gravid status), and/or blood (engorged: total or partial vs. unfed) in the abdomen was recorded (Table 1).

\section{DNA extraction, PCR amplification and DNA sequencing}

For each female sand fly, the remainder of the body (minus genitalia) was used as the source of DNA, extracted using the Citogene ${ }^{\bullet}$ Cell and Tissue kit (Citomed, Portugal) following the manufacturer's instructions, with the exception that the maceration of the insect's tissues was carried out with a piston pellet, and the final elution volume was $30 \mu \mathrm{l}$.

The PCR amplification of the internal transcribed spacer 1 (ITS-1) of the ribosomal operon of Leishmania was performed using the LITSR and L5.8S primers generating amplicons with 300-350 bp [8]. A positive control containing L. infantum DNA (MHOM/PT/88/ IMT151) and a negative control without DNA template were included. To identify the origin of the blood meal of engorged females, the modified vertebrate-universal specific primers (cytB1-F and cytB2-R) were used to amplify a $350 \mathrm{bp}$ segment of the host mitochondrial cytochrome $b$ gene (cyt-b) [9]. PCR amplifications were performed in a $25 \mu \mathrm{l}$ final volume containing $12.5 \mu \mathrm{l}$ of NZYTaq $2 \times$ Green Master Mix (Nyztech, Portugal), $1 \mu \mathrm{l}$ of each primer $(10 \mathrm{pmol})$ and $2 \mu \mathrm{l}$ of template DNA. The cycling profile used for the amplification of ITS-1 sequences included an initial denaturation step at $95^{\circ} \mathrm{C}$ for $2 \mathrm{~min}$, followed by 32 repeats of $95^{\circ} \mathrm{C}-20 \mathrm{sec}, 53^{\circ} \mathrm{C}-30 \mathrm{sec}, 72^{\circ} \mathrm{C}-1$ min followed by a final extension step at $72^{\circ} \mathrm{C}-6 \mathrm{~min}$, while the preparation of $c y t-b$ PCR products was carried out starting from $95^{\circ} \mathrm{C}$ for $5 \mathrm{~min}$, followed by 40 cycles of $94^{\circ} \mathrm{C}-1 \mathrm{~min}$, $55^{\circ} \mathrm{C}-1 \mathrm{~min}, 72^{\circ} \mathrm{C}-1 \mathrm{~min}$ followed by $72^{\circ} \mathrm{C}-7 \mathrm{~min}$. Both amplicons were visualized under UV illumination after their resolution by conventional electrophoresis on $1.5 \%$ agarose gels stained with Greensafe premium ${ }^{\circ}$ (Nzytech, Portugal), using a 100 bp DNA ladder as a molecular weight marker. PCR products were purified with a High Pure PCR Product Purification Kit (Roche ${ }^{\circ}$ Mannheim, Germany) according to the manufacturer's instructions. Subsequently, purified products were sent to LIGHTrun ${ }^{\text {TM }}$ Sequencing Service (GATC-biotech, Germany) for direct sequencing by Sanger's method with the same primers used for DNA amplification.

\section{DNA sequence analyses}

The identity of the feeding host (species level), carried out on the basis of the analysis of the obtained $c y t-b$ sequences, was determined according to the closest BLASTn match (identity $\geq 99 \%$ ) to a homologous sequence deposited at GenBank. The sequences obtained in the course of this work were deposited at DNA Data Bank of Japan (DDBJ) (http://www.DDBJ.nig.ac.jp).

Restriction profile was obtained by virtual digestion for ITS-1 sequence by using the Restriction Mapper (version 3 available online at http://www.restrictionmapper. $\operatorname{org} /)$. 
Table 1 Sand fly specimens collected according to the capture method and their positivity to Leishmania spp.

\begin{tabular}{|c|c|c|c|c|c|c|c|c|c|c|}
\hline \multirow[b]{2}{*}{ Sand fly species } & \multicolumn{2}{|c|}{ CDC light traps } & \multicolumn{2}{|c|}{ Sitcky papers } & \multicolumn{3}{|l|}{ Total } & \multicolumn{3}{|l|}{ Females } \\
\hline & Females & Males & Females & Males & Females & Males & Females + Males & Blood fed & Gravid & Positive \\
\hline Phlebotomus ariasi & 34 & 8 & 0 & 4 & 34 & 12 & 46 & 3 & 2 & 0 \\
\hline Phlebotomus perniciosus & 372 & 305 & 59 & 806 & 431 & 1111 & 1542 & 49 & 32 & 1 \\
\hline Phlebotomus papaptasi & 1 & & & & 1 & & 1 & & 1 & \\
\hline Phlebotomus sergenti & 27 & 26 & 27 & 94 & 54 & 120 & 174 & 1 & 1 & 0 \\
\hline Sergentomyia minuta & 212 & 149 & 1655 & 1192 & 1867 & 1341 & 3208 & 25 & 49 & 2 \\
\hline Total & 646 & 488 & 1741 & 2096 & 2387 & 2584 & 4971 & 78 & 85 & 3 \\
\hline
\end{tabular}

Phylogenetic relationships were inferred from ITS-1 nucleotide sequence alignments produced with the MAFFT multiple alignment program using a combination of the Q-INS-i and E-INS-i alignment options [10]. Phylogenetic tree construction was carried out using a Maximum Likelihood (ML) approach, and the Kimura's 2-P (K2P) evolutionary model, also assuming $\Gamma$ distributed substitution rates among sites, as indicated by Mega6 [11] and as defined by the Akaike information criterion. Alternatively, an empirically defined model $(G T R+\Gamma+I)$ was also used. The topological robustness of the obtained trees was assessed by bootstrapping, using 1000 resampling of the original alignment data. The final trees were manipulated for display using FigTree v.1.2.2. (available at http://tree. bio.ed.ac.uk/software/figtree/). NeighborNet networks $(\mathrm{NNn})$ were constructed using the same distance matrix using Splits Tree4 software [12]; software available at http://www.splitstree.org/). Mean genetic distance values were calculated with the K2P formula, using Mega6 [11].

\section{Results}

\section{Morphological identification of sand flies}

$S$. minuta was the most prevalent species totaling a number of 3,208 specimens (64.53\%), followed by $P$. perniciosus with 1,542 specimens (31.02\%). Phlebotomus sergenti $(174 ; 3.50 \%), P$. ariasi $(46 ; 0.93 \%)$ and one $P$. papatasi female $(0.02 \%)$ were also collected. Eighty five females (2 P. ariasi, 1 P. papatasi, 32 P. perniciosus, 1 P. sergenti and $49 \mathrm{~S}$. minuta) were gravid.

\section{Leishmania DNA detection, sequencing, and phylogenetic} inference analysis

Leishmania DNA was detected in three apparently unfed females $(0.13 \%)$ identified as $P$. perniciosus $(\mathrm{n}=1)$ and in $S$. minuta $(\mathrm{n}=2)$. The three positive females were collected in peridomestic biotopes (i.e. P. perniciosus was collected in a horse stable, and $S$. minuta were collected in a cattle pen and close to a kennel, respectively). The three ITS-1 obtained sequences were submitted to DDBJ (DDBJ accession numbers: LC028233 to LC028235). PCR product obtained from $P$. perniciosus had a similar size as $L$. infantum control while the PCRs products from both $S$. minuta were slightly bigger (data not shown). Furthermore, a HaeIII restriction profile characteristic of L. infantum (184 bp, $72 \mathrm{bp}$ and $55 \mathrm{bp}$ ) was obtained after virtual digestion of the ITS-1 sequence obtained from the positive DNA control as well as from P. perniciosus. Finally, sequence homology searches using BLASTn (megablast search option) revealed $>99 \%$ identity with $L$. infantum, L. chagasi or L. donovani (E-values $\left.=\mathrm{e}^{-154}\right)$, and a sequence coverage $>94 \%$. Curiously, however, species assignment to the ITS-1 sequences amplified from $S$. minuta could not be carried out on the basis of nucleotide sequence homology search results. In this case the 15 best matches obtained with BLASTn (megablast) revealed $>93 \%$ sequence identity ( $>95 \%$ sequence coverage and E-values $<\mathrm{e}^{-122}$ ) with only Leishmania sequences of Chinese origin referred to as Leishmania sp. [13], indicating relatively low identity with any sequence references already deposited in the sequence databases. Virtual HaeIII restriction profiles of the ITS-1 sequences amplified from S. minuta (strains 5277 and 3400) were characterized by three DNA fragments (<193 bp, $89 \mathrm{bp},<54 \mathrm{bp}$ ), which were found to be similar, though not identical, to the virtual HaeIII profiles determined for the Chinese Leishmania sp. sequences ( $<210 \mathrm{bp}$, $87 \mathrm{bp},<43 \mathrm{bp}$ ) mentioned above.

Definition of the species status of the obtained ITS-1 sequences was further pursued on the basis of phylogenetic analyses, along with others directly downloaded from the public database, and used as references (Table 2). The use of the suggested evolutionary model $(\mathrm{K} 2 \mathrm{P}+\mathrm{\Gamma})$ or a more robust one $(\mathrm{GTR}+\mathrm{\Gamma}+\mathrm{I})$, empirically defined by the user, resorted in phylogenetic trees with identical topologies as that shown in Figure 1 (data not shown).

One of the sequences obtained in this study (strain 1704), amplified from $P$. perniciosus, was found to segregate in a large monophyletic cluster that included $L$. infantum, L. donovani, L. archibaldi and L. chagasi (Figure 1), characterized by low genetic variability (average genetic distance of $0.2 \%$ ). On the other hand, the remainder two ITS-1 sequences (strains 5277 and 3400), 
Table 2 Nucleotide reference sequences used in this work

\begin{tabular}{|c|c|c|c|}
\hline Species* & Strain/isolate/haplotype & Origin/host & Accession number \\
\hline Leishmania donovani & MHOM/KE/83/NLB189 & Kenya/Human & AJ634374 \\
\hline Leishmania donovani & MHOM/SD/93/9S & Sudan/Human & AJ634372 \\
\hline Leishmania donovani & MHOM/LK/2002/L60C & Sri Lanka/Human & AM901447 \\
\hline Leishmania donovani & MHOM/LK/2002/L60b & Sri Lanka/Human & AM901448 \\
\hline Leishmania archibaldi & MHOM/SD/93/GE & Sudan/Human & AJ634357 \\
\hline Leishmania archibaldi & MHOM/SD/97/LEM3429 & Sudan/Human & AJ634358 \\
\hline Leishmania archibaldi & MHOM/SD/97/LEM3463 & Sudan/Human & AJ634359 \\
\hline Leishmania donovani & MHOM/SU/84/LEM0946 & Soviet Union/Human & HG512918 \\
\hline Leishmania donovani & MCAN/MA/2002/AD3 & Morocco/Canine & AM901453 \\
\hline Leishmania donovani & MHOM/IQ/1981/SUKKAR2 & Iraq/Human & AM901452 \\
\hline Leishmania donovani & MHOM/IN/1983/CHANDIGARH & India/Human & AM901449 \\
\hline Leishmania infantum & MCAN/UZ/2007/LRC-L1309 & Uzbekistan/Canine & FN398341 \\
\hline Leishmania infantum & MHOM/BR/2007/JFF BM & Brazil/Human & FN398343 \\
\hline Leishmania infantum & MHOM/IT/93/ISS800 & Italy/Human & AJ634354 \\
\hline Leishmania infantum & MHOM/PT/00/IMT260 & Portugal/Human & AJ634344 \\
\hline Leishmania infantum & MHOM/MT/85/BUCK & Malta/Human & AJ634350 \\
\hline Leishmania infantum & $\mathrm{MHOM} / \mathrm{SD} / 93 / 452 \mathrm{BM}$ & Sudan/Human & AJ634371 \\
\hline Leishmania chagasi & MHOM/BR/85/M9702 & Brazil/Human & AJ000306 \\
\hline Leishmania chagasi & MHOM/PA/79/WR317 & Panama/Human & AJ000305 \\
\hline Leishmania tropica & MHOM/IL/01/LRC-L838 & Israel/Human & FN677341 \\
\hline Leishmania tropica & MHOM/EG/90/LPN65 & Egypt/Human & HG512927 \\
\hline Leishmania tropica & MHOM/PS/01/ISL590 & Palestine*/Human & FN677345 \\
\hline Leishmania tropica & MHOM/YE/86/LEM1015 & Yemen/Human & HG512919 \\
\hline Leishmania tropica & MHOM/TN/88/TAT3 & Tunisia/Human & AJ300485 \\
\hline Leishmania tropica & IHAM/GH/2007/KLE-18 & Ghana/Sergentomyia hamoni & AB787190 \\
\hline Leishmania aethiopica & MHOM/ER/2009/7457 & Eritrea/Human & FN252411 \\
\hline Leishmania aethiopica & MHOM/KE/71/KPS-H2 & Kenya/Human & HG512908 \\
\hline Leishmania turanica & KD85001 & Uzbekistan/Rhombomys opimus & AJ272378 \\
\hline Leishmania turanica & KL3 & Kazakhstan/Rhombomys opimus & AJ272382 \\
\hline Leishmania gerbilli & MRHO/UZ/87/KD-87555 & Uzbekistan/Rhombomys opimus & AJ300486 \\
\hline Leishmania major & MTAT/KE//NLB089A & Kenya/ND & AJ300482 \\
\hline Leishmania major & MHOM/UZ/02/17h & Uzbekistan/Human & FN677357 \\
\hline Leishmania major & MHOM/BF/2004/REN04-8 & Burkina Faso/Human & HG512963 \\
\hline Leishmania major & MHOM/JO/90/JH39 & Jordan/Human & HG512945 \\
\hline Leishmania major & MHOM/TN/97/LPN162 & Tunisia/Human & FN677342 \\
\hline Leishmania major & MHOM/DZ/89/LIPA228 & Algeria/Human & HG512924 \\
\hline Leishmania mexicana & MHOM/PE/02/LH2312 & Peru/Human & HG512965 \\
\hline Leishmania mexicana & MHOM/EC/90/LM & Ecuador/Human & HG512934 \\
\hline Leishmania amazonensis & MHOM/BR/73/M2269 & Brazil/Human & DQ182536 \\
\hline Leishmania amazonensis & IFLA/BR/67/PH8 & Brazil/ND & AF339753 \\
\hline Leishmania braziliensis & MHOM/PE/2003/LH2920 & Peru/Human & FN398337 \\
\hline Leishmania braziliensis & MHOM/BR/00/LTB300 & Brazil/Human & FN398338 \\
\hline Leishmania peruviana & MHOM/PE/2006/LH3667 & Peru/Human & FN398340 \\
\hline Leishmania peruviana & MHOM/PE/1990/HB86 & Peru/Human & FN398339 \\
\hline
\end{tabular}


Table 2 Nucleotide reference sequences used in this work (Continued)

\begin{tabular}{|c|c|c|c|}
\hline Leishmania guyanensis & MHOM/BR/2002/NMT-RBO013 & Brazil/Human & FN398331 \\
\hline Leishmania guyanensis & MHOM/PE/2006/LH3635 & Peru/Human & FN398332 \\
\hline Leishmania panamensis & Isolate 18, clone 4 & ND/Human & FJ948442 \\
\hline Leishmania sp. & MHOM/CN/80/XJ801 & P.R.China/Human & HQ830357 \\
\hline Leishmania sp. & MHOM/CN/89/GS5 & P.R.China/Human & HQ830360 \\
\hline Leishmania sp. & MHOM/CN/90/SC10H2 & P.R.China/Human & HQ830352 \\
\hline Leishmania sp. & MHOM/CN/86/SC6 & P.R.China/Human & HQ830356 \\
\hline Leishmania sp. & $\mathrm{MHOM} / \mathrm{CN} / 90 / \mathrm{SC} 10 \mathrm{H} 2$ & P.R.China/Human & HM130601 \\
\hline Leishmania sp. & MCAN/CN/60/GS1 & P.R.China/Canine & HM130600 \\
\hline Leishmania sp. & MHOM/GS6/CHN/SCgq & P.R.China/Human & HM130599 \\
\hline Leishmania sp. & MCAN/CN/86/SC9 & P.R.China/Canine & HQ830359 \\
\hline Leishmania sp. & MHOM/CN/83/GS2 & P.R.China/Human & HM130603 \\
\hline Leishmania sp. & $\mathrm{MHOM} / \mathrm{GS} 5 / \mathrm{CHN} / \mathrm{SCH} 2 \mathrm{~g}$ & P.R.China/Human & HM130602 \\
\hline Leishmania sp. & MHOM/SC11/CHN/SCgz & P.R.China/Human & HM130606 \\
\hline Leishmania sp. & MHOM/CN/84/JS1 & P.R.China/Human & HM130605 \\
\hline Leishmania sp. & MHOM/CN/84/SD1 & P.R.China/Human & HM130604 \\
\hline Leishmania sp. & MHOM/CN/89/GS6 & P.R.China/Human & HQ830355 \\
\hline Leishmania sp. & MHOM/CN/90/SC11 & P.R.China/Human & HQ830361 \\
\hline
\end{tabular}

*Species as defined by the depositors; Israel: Occupied Palestinian Territories; P. R. China: People's Republic of China; ND: not defined.

amplified from S. minuta, were found to locate in a bootstrap-supported (99\%) assemblage of multiple reference sequences of Chinese origin, merely defined as Leishmania sp. [13], and that included a multitude of Leishmania sequences from human and canine origin, with an average genetic distance of $2.6 \%$ (ranging from $0 \%$ to $8.0 \%$ ), indicating considerably higher genetic variability than that associated with the L. infantum/L. donovani/L. archibaldi/L. chagasi cluster. Similar conclusions were achieved when, instead of assuming a strict treelike evolution, the phylogenetic relationships between ITS-1 sequences were represented as a NNn (Figure 2).

\section{Vertebrate DNA detection in female sand flies}

A total of 78 engorged female sand flies (3 P. ariasi, 49 $P$. perniciosus, $1 P$. sergenti and $25 S$. minuta) were tested to determine the vertebrate host source of the blood meal. A positive PCR amplification result was obtained for 43 of the collected specimens. After DNA sequencing of the amplified partial $c y t-b$ sequences, the origin of $30(69.77 \%)$ blood-meals was identified (Table 3$)$ on the basis of the closest sequence matches, as defined by BLASTn sequence homology searches (>99\% identity with deposited at the GenBank/EMBL/DDBJ public databases).

\section{Discussion}

Phlebotomine sand flies are distributed in all countries around the Mediterranean basin, turning both human populations and domestic animals living in these areas into potential targets to sand fly-borne diseases such as leishmaniasis. Therefore, knowledge on the host preferences of sand flies under natural conditions is essential not only to understand their vectorial role, but also as a means to identify potential reservoir hosts. In this work, we detected Leishmania DNA and evaluated blood meal sources of fed females sand flies captured in southern Portugal, where zoonotic leishmaniasis is known to be endemic [2].

Similarly to what has been observed by others [14-19] the blood meal analysis of the engorged $P$. perniciosus revealed that this species fed on a broad variety of vertebrates hosts (i.e. horses, cattle, sheep, pigs, rabbits and chickens) highlighting its opportunistic feeding behaviour. Interestingly, no dog or human blood was detected in blood-fed $P$. perniciosus, despite the fact that it has been clearly defined as a proven vector of L. infantum in the Algarve region [19-21]. The apparent absence of $P$. perniciosus feeding on dogs and humans might indicate that in the sampled biotopes, neither of them were the main blood sources for this sand fly species due to the presence of other larger sized vertebrates (e.g. horses) and/or present in greater numbers (i.e. chicken, rabbits), making them easier targets.

In addition, Leishmania infantum DNA was detected in one unfed $P$. perniciosus specimen. Assignment of species status for the 1704 ITS-1 sequence could not be clearly carried out solely based on phylogenetic tree analysis due to the low genetic variability of the ITS-1 sequences that define the $L$. donovani complex [22] (that 


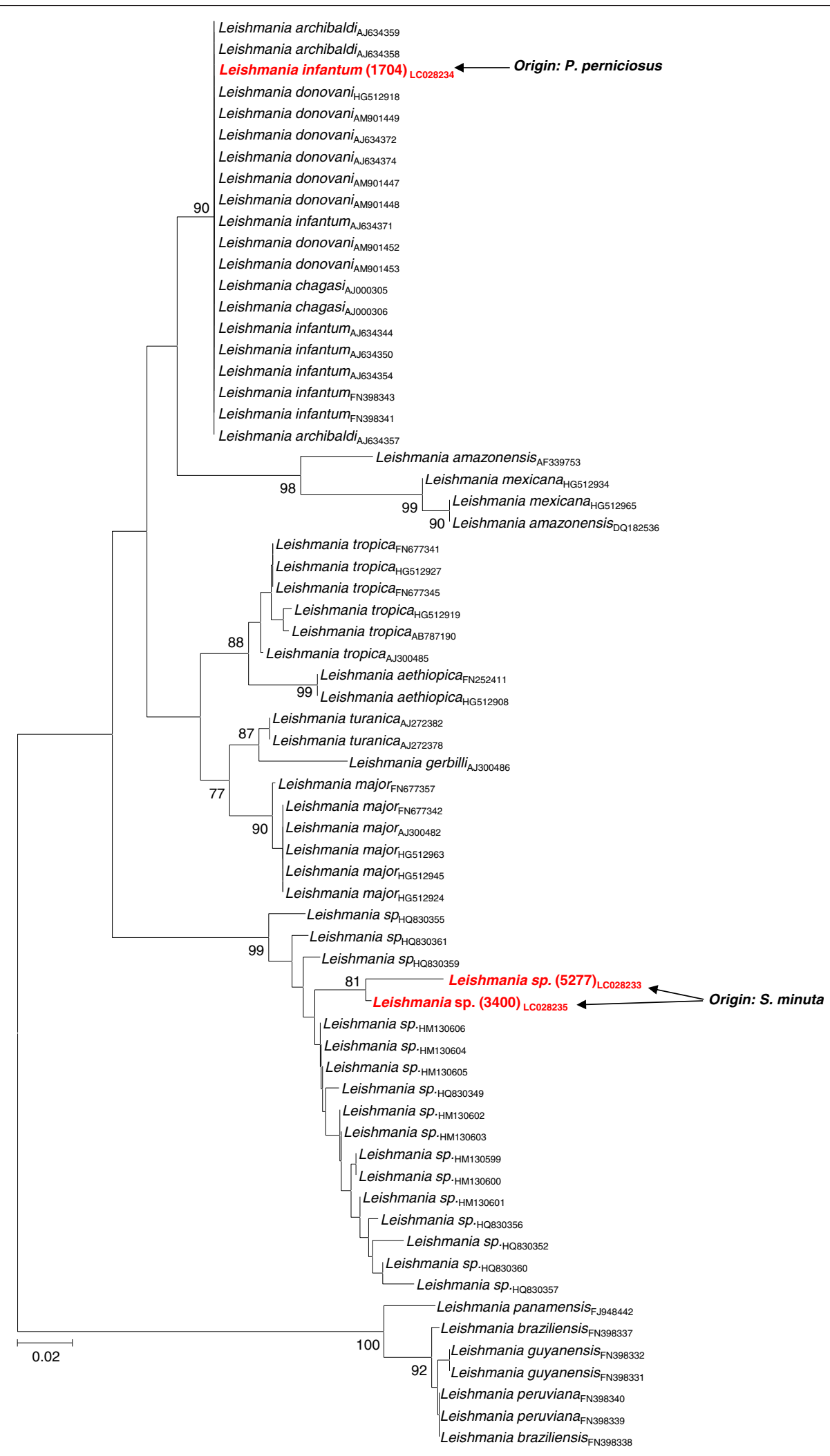

Figure 1 Maximum likelihood phylogenetic tree (midpoint rooted) of Leishmania ITS-1 sequences amplified from phlebotomine sand flies collected in Portugal. The percentages of significant ( $\geq 77 \%)$ bootstrap values of 1000 resamplings of the original data are indicated at specific branch-nodes. The size bar indicates 0.02 substitutions per site. 


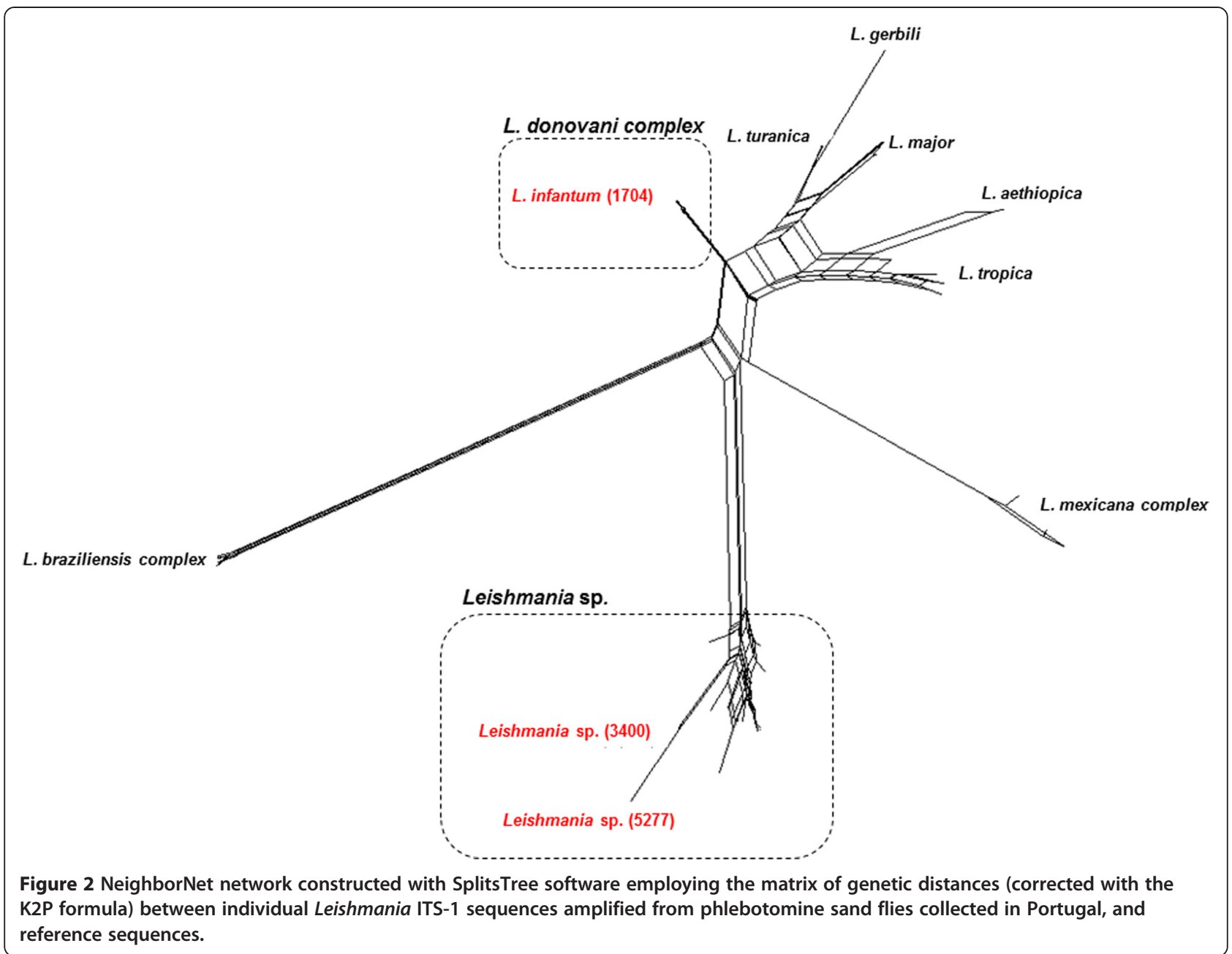

include $L$. infantum, L. donovani, $L$. archibaldi and $L$. chagasi, precluding a clear resolution of this genetic cluster, as previously observed [13]. Nevertheless, the ITS-1 amplicon size and virtual HaeIII restriction profile obtained for the 1704 sequence amplified from $P$. perniciosus were compatible with it corresponding to L. infantum, and reinforces the maintenance of this sand fly species as vector of L. infantum in southern Portugal [19-21].

Sand flies of the Sergentomyia genus, which is widely distributed throughout the Old World, are proven vectors of reptile Leishmania species [23]. It is generally accepted that most of Sergentomyia species are not anthropophilic, and as a consequence cannot transmit either Leishmania or any other pathogens to humans. However, in the present study, apart from detecting Tarentola mauritanica (a reptile widely distributed around the Mediterranean area $[23,24])$ DNA in one engorged S. minuta, human DNA was also amplified in four specimens corroborating that at least some Sergentomyia species disclose sporadic/opportunistic anthropophilic feeding-behaviour [25,26]. Furthermore, Leishmania sp. DNA was detected in two unfed $S$. minuta females, which unambiguously allocated with references within a cluster of Chinese Leishmania sp. previously isolated from humans and canine leishmaniasis cases [13]. While phylogenetic tree reconstruction and $\mathrm{NNn}$ analyses showed that the two ITS-1 sequences amplified from $S$. minuta (strains 5277 and 3400) clearly segregated away from all the others in a genetically consistent assemblage of Leishmania strains, in this case species assignment was limited by the unavailability of well characterized reference strains. However, despite the inability to clearly define the species of origin of the obtained sequences using phylogenetic analyses, the detection of Leishmania DNA phylogenetically related to those considered pathogenic to humans and dogs in China [13] was somewhat unexpected.

According to Yang et al. [13], the above mentioned Leishmania strains of Chinese origin belonged to an undefined species, that was found to be genetic divergent from any of the known New and Old World Leishmania, 
Table 3 Identification of sand fly blood meal sources

\begin{tabular}{|c|c|c|c|c|c|c|c|c|c|c|c|}
\hline \multirow{2}{*}{$\begin{array}{l}\text { Sand fly host } \\
\text { Horse (Equus caballus) }\end{array}$} & \multirow{2}{*}{$\begin{array}{l}\text { P. ariasi } \\
0\end{array}$} & \multirow{2}{*}{$\begin{array}{l}\text { P. perniciosus } \\
12\end{array}$} & \multirow{2}{*}{$\begin{array}{l}\text { S. minuta } \\
0\end{array}$} & \multirow{2}{*}{$\begin{array}{l}\text { Blast identity for the blood meal } \\
99-100 \%\end{array}$} & \multicolumn{7}{|c|}{ DDBJ accession no. } \\
\hline & & & & & AB985687 & AB985693-97 & AB985699 & AB985703 & AB985708 & AB985711 & AB985714 \\
\hline Chicken (Gallus gallus) & 0 & 5 & 0 & $99-100 \%$ & & & AB985704 & AB985705 & AB985710 & AB985713 & AB985715 \\
\hline Human (Homo sapiens) & 0 & 0 & 4 & $99-100 \%$ & & & & AB985688 & AB985689 & AB985698 & AB985712 \\
\hline Rabbit (Oryctolagus cuniculus) & 0 & 3 & 0 & $99 \%$ & & & & & AB985690 & AB985700 & AB985709 \\
\hline Pig (Sus scrofa) & 0 & 2 & 0 & 99 & & & & & & AB985707 & AB985716 \\
\hline Cattle (Bos taurus) & 1 & 1 & 0 & 99 & & & & & & AB985702 & AB985706 \\
\hline Sheep (Ovis aries) & 0 & 1 & 0 & 99 & & & & & & & AB985701 \\
\hline Lizard (Tarentola mauritanica) & 0 & 0 & 1 & 99 & & & & & & & AB985692 \\
\hline Total & 1 & 24 & 5 & & & & & & & & \\
\hline
\end{tabular}


on the basis of ITS-1 sequence analysis. Similar results were obtained when kinetoplast cytochrome oxidase II (COII; [27]) or CYT-b coding sequences [28] amplified from these same strains were analysed. Interestingly, both phylogenetic inference reconstruction studies revealed that the Chinese Leishmania sp. isolates were most closely related to the lizard-infecting $L$. tarentolae. Unfortunately, in the present study it was not possible to evaluate if the two Leishmania sp. detected in S. minuta were genetic related to this reptile Leishmania species, as no ITS-1 sequences of $L$. tarentolae have yet been deposited in DNA sequence databases for public access. On the other hand, exhaustion of the DNA extracts on which the analysis presented in this report was based ruled out any possibility of generating $c y t-b$ and/or coII sequence data. Nevertheless, and taking into account the results obtained with $c y t$-b/coII $[27,28]$, in the near future it will be important to analyse more of these Leishmania parasites obtained from both vertebrate (including reptiles) and invertebrate infected hosts for assessment of the parasite species as well as to determine their clinical significance, and estimate the potential risk their endemic establishment in Portugal/Europe. Ideally, should the laboratory settings allow it, further genetic analysis-based studies should be supported, as much as possible, by sequence datasets combining information from multiple genetic loci, so as to tentatively increase the phylogenetic signal, and achieve a better resolution of the observed genetic clusters, including the L. donovani complex [29].

Based upon literature reviews, a consideration of the role of Sergentomyia in the circulation of mammalian leishmaniasis becomes apparent as Leishmania DNA has been identified in several species. These include the molecular detection of L. major in S. sintoni in Iran [30], $S$. garnhami in Kenya [31], S. darlingi in Mali [25], and $S$. minuta in Portugal [32]. Furthermore L. donovani has been detected in S. babu in India [33], L. infantum in $S$. dubia, S. magna and S. schewtzi in Senegal [34], and $L$. siamennsis in S. gemmea in Thailand [35]. Finally, more recently, $L$. tropica has been found in S. ingrami and $S$. hamoni in Ghana [26]. Nevertheless, PCR positivity alone should not be used for incrimination of Sergentomyia sand flies as Leishmania vectors since the detection of DNA does not give any information about the parasites' viability or its presence as virulent metacyclic promastigotes [36,37]. In fact, and although $L$. infantum DNA had been detected in S. schwetzi from Senegal [34], the refractoriness of this African species to some Leishmania species infecting humans (including L. donovani, L. infantum and $L$. major) has also been recently demonstrated [38]. In any case, the refractoriness of this particular Sergentomyia species does not necessarily extend to the whole of the genus. In this line of reasoning, the competence and permissiveness of the different species from Phlebotomus spp. to different Old World Leishmania has also been observed [39]. As L. major DNA had previously been detected in one $S$. minuta captured in the same region [32], together with the detection in this study of both human and Leishmania sp. DNA in this species, it would be important to determine if S. minuta fulfils the criteria that support its incrimination as vector for this parasite, and that include (i) the isolation of metacyclic promastigotes from the digestive tubes of field-collected specimens; and (ii) the experimental demonstration of its capacity to transmit Old World Leishmania species with medical and veterinarian importance as a result of blood-feeding on mammals.

\section{Conclusion}

The apparent anthropophilic behavior of S. minuta together with the detection of Leishmania sp. DNA highlight the need to determine the role played by this sand fly species in the transmission of pathogenic Leishmania to humans. In addition, our data confirms that $P$. perniciosus is an opportunistic feeder and suggest that is responsible for the maintenance of L. infantum in sourthern Portugal. Altogether, the obtained results reinforce the need for ongoing surveillance with systematic epidemiologic surveys on Leishmania vectors so as to investigate the transmission, distribution and spread of infections by Leishmania species.

\section{Competing interests}

The authors declare that they have no competing interests.

\section{Authors' contributions}

CM planned the study, collected samples, performed DNA extraction and wrote the manuscript; RP performed DNA sequence analysis and revised the manuscript; JMC performed molecular analyses; FF collected samples and performed DNA extraction; MOA performed morphological identification and revised the manuscript; LC supervised the study and reviewed the manuscript. All authors read and approved the final manuscript.

\section{Acknowledgements}

This work was supported by EU grant FP7-261504 EDENext, and is catalogued by the EDENext Steering Committee as EDENext306 (http://www.edenext.eu). The contents of this publication are the sole responsibility of the authors and do not necessarily reflect the views of the European Commission. We would like to thank to all the inhabitants that allowed the placement of the CDC and sticky traps in their properties. CM (SFRH/BPD/44082/2008) holds a scholarship from Fundação para a Ciência e a Tecnologia, Ministério da Educação e Ciência, Portugal. The work of C. Maia was done under the frame of EurNegVec COST Action TD1303.

Publication of the CVBD10 thematic series has been sponsored by Bayer HealthCare - Animal Health division.

\section{Author details}

${ }^{1}$ Unidade de Parasitologia Médica, Global Health and Tropical Medicine (GHTM), Instituto de Higiene e Medicina Tropical (IHMT), Universidade Nova de Lisboa (UNL), Lisbon, Portugal. Unidade de Microbiologia Médica, GHTM, IHMT-UNL, Lisbon, Portugal. ${ }^{3}$ Departamento de Ciências Biomédicas e Medicina, Universidade do Algarve, Faro, Portugal.

Received: 15 January 2015 Accepted: 6 March 2015

Published online: 23 March 2015 


\section{References}

1. Dujardin J, Campino L, Cañavate C, Dedet J, Gradoni L, Soteriadou K, et al. Spread of vector-borne diseases and neglect of Leishmaniasis, Europe. Emerg Infect Dis. 2008;14:1013-8.

2. Campino L, Maia C. Epidemiology of leishmaniases in Portugal. Acta Med Port. 2010;23:859-64.

3. Campino L, Pratlong F, Abranches P, Rioux J, Santos-Gomes G, Alves-Pires C, et al. Leishmaniasis in Portugal: enzyme polymorphism of Leishmania infantum based on the identification of 213 strains. Trop Med Int Health. 2006:11:1708-14.

4. $\quad$ Pires C. Les phlébotomes du Portugal I- Infestation naturelle de Phlebotomus ariasi Tonnoir, 1921 et Phlebotomus perniciosus Newstead, 1911, par Leishmania dans le foyer zoonotique de Arrábida (Portugal). Ann Parasitol Humaine Comparee. 1984;59:521-4

5. Censos. Resultados Preliminares 2011. Instituto Nacional de Estatística. Available from http://www.ine.pt/xportal/xmain? xpid=INE\&xpgid=ine_indicadores\&indOcorrCod=0005889\&selTab=tab0. Acessed 12 Jan 2015

6. Franco J. Zonas fitogeográficas predominantes de Portugal Continental. Anais ISA. 1994:44:39-56.

7. Pires C. Contribuição para o conhecimento da distribuição e bioecologia dos flebótomos em Portugal (Díptera: Psychodidae). B Soc Port Ciên Nat. 1979;19:197-210.

8. Schonian G, Nazereddin A, Dinse N, Schweynoch C, Shallig H, Presber W, et al. PCR diagnosis and characterization of Leishmania in local and imported clinical samples. Diagn Microbiol Infect Dis. 2003;47:349-58.

9. Svobodova M, Alten B, Zidkova L, Dvorak V, Hlavacková J, Mysková J. Cutaneous leishmaniasis caused by Leishmania infantum transmitted Phlebotomus tobbi. Int J Parasitol. 2008;39:251-6.

10. Katoh K, Toh H. Recent developments in the MAFFT multiple sequence alignment program. Brief Bioinform. 2008;9:286-98.

11. Tamura K, Stecher G, Peterson D, Filipski A, Kumar S. MEGA6: Molecular Evolutionary Genetics Analysis version 6.0. Mol Biol Evol. 2013;30:2725-9.

12. Huson DH, Bryant D. Application of phylogenetic networks in evolutionary studies. Mol Biol Evol. 2006;23:254-67.

13. Yang BB, Guo XG, Hu XS, Zhang JG, Liao L, Chen DL, et al. Species discrimination and phylogenetic inference of 17 Chinese Leishmania isolates based on internal transcribed spacer 1 (ITS1) sequences. Parasitol Res. 2010;107:1049-65.

14. Bongiorno G, Habluetzel A, Khoury C, Maroli M. Host preferences of phlebotomine sandflies at a hypoendemic focus of canine leishmaniasis in central Italy. Acta Trop. 2003:88:109-16.

15. Rossi E, Bongiorno G, Ciolli E, Di Muccio T, Scalone A, Gramiccia M, et al. Seasonal phenology, host-blood feeding preferences and natural Leishmania infection of Phlebotomus perniciosus (Diptera, Psychodidae) in a high-endemic focus of canine leishmaniasis in Rome province Italy. Acta Trop. 2008:105:158-65.

16. Branco S, Alves-Pires C, Maia C, Cortes S, Cristovão J, Gonçalves L, et al. Entomological and ecological studies in a new potential zoonotic leishmaniasis focus in Torres Novas municipality, central region, Portugal. Acta Trop. 2013;125:339-48.

17. Jaouadi K, Haouas N, Chaara D, Boudabous R, Gorcii M, Kidar A, et al. Phlebotomine (Diptera, Psychodidae) blood meal sources in Tunisian cutaneous leishmaniasis foci: could Sergentomyia minuta, which is not an exclusive herpetophilic species, be implicated in the transmission of pathogens? Ann Entomol Soc Am. 2013;106:79-85.

18. Jiménez M, González E, Iriso A, Marco E, Alegret A, Fúster F, et al. Detection of Leishmania infantum and identification of blood meals in Phlebotomus perniciosus from a focus of human leishmaniasis in Madrid, Spain. Parasitol Res. 2013;112:2453-9.

19. Maia C, Dionísio L, Afonso MO, Neto L, Cristóvão JM, Campino L. Leishmania infection and host-blood feeding preferences of phlebotomine sandflies and canine leishmaniasis in an endemic European area, the Algarve Region in Portugal. Mem Inst Oswaldo Cruz. 2013;108:481-7.

20. Alves-Pires C, Campino L, Afonso MO, Santos-Gomes G, Dedet JP, Pratlong F. Les phlébotomes du Portugal. X. Infestation naturelle de Phlebotomus perniciosus par Leishmania infantum MON-1 en Algarve. Parasite. 2001:8:374-5.

21. Maia C, Afonso MO, Neto L, Dionísio L, Campino L. Molecular detection of Leishmania infantum in naturally infected Phlebotomus perniciosus from Algarve region, Portugal. J Vector Borne Dis. 2009;46:268-72.
22. Lukes J, Mauricio IL, Schönian G, Dujardin JC, Soteriadou K, Dedet JP, et al. Evolutionary and geographical history of the Leishmania donovani complex with a revision of current taxonomy. Proc Natl Acad Sci U S A. 2007:104:9375-8

23. Bates P. Transmission of Leishmania metacyclic promastigotes by phlebotomine sand flies. Int J Parasitol. 2007;37:1097-106.

24. Alves-Pires C, Campino L, Janz JG, Afonso MO. Os flebótomos de Portugal. XIV. Os vectores de leishmanioses no foco zoonótico do sotavento Algarvio. Acta Parasitol Port. 2008;15:38-52

25. Berdjane-Brouk Z, Koné AK, Djimdé AA, Charrel RN, Ravel C, Delaunay P, et al. First detection of Leishmania major DNA in Sergentomyia (Spelaeomyia) darlingi from cutaneous leishmaniasis foci in Mali. PLoS One. 2012;7:e28266.

26. Nzelu CO, Kato H, Puplampu N, Desewu K, Odoom S, Wilson MD, et al. First detection of Leishmania tropica DNA and Trypanosoma species in Sergentomyia sand flies (Diptera: Psychodidae) from an outbreak area of cutaneous leishmaniasis in Ghana. PLoS Negl Trop Dis. 2014;8:e2630.

27. Cao DP, Guo XG, Chen DL, Chen JP. Species delimitation and phylogenetic relationships of Chinese Leishmania isolates reexamined using kinetoplast cytochrome oxidase II gene sequences. Parasitol Res. 2011;109:163-73.

28. Yang BB, Chen DL, Chen JP, Liao L, Hu XS, Xu JN. Analysis of kinetoplast cytochrome $b$ gene of 16 Leishmania isolates from different foci of China: different species of Leishmania in China and their phylogenetic inference. Parasit Vectors. 2013;6:32

29. Van der Auwera G, Ravel C, Verweij JJ, Bart A, Schönian G, Felger I. Evaluation of four single-locus markers for Leishmania species discrimination by sequencing. J Clin Microbiol. 2014;52:1098-104.

30. Parvizi P, Amirkhani A. Mitochondrial DNA characterization of Sergentomyia sintoni populations and finding mammalian Leishmania infections in this sandfly by using ITS-rDNA gene. Iranian J Vet Res. 2008;9:9-18.

31. Mutinga MJ, Massamba NN, Basimike M, Kamau CC, Amimo FA, Onyido AE, et al. Cutaneous leishmaniasis in Kenya: Sergentomyia garnhami (Diptera Psychodidae), a possible vector of Leishmania major in Kitui District: a new focus of the disease. East Afr Med J. 1994:71:424-8.

32. Campino L, Cortes S, Dionísio L, Neto L, Afonso MO, Maia C. The first detection of Leishmania major in naturally infected Sergentomyia minuta in Portugal. Mem Inst Oswaldo Cruz. 2013;108:516-8.

33. Mukherjee S, Hassan MQ, Ghosh A, Ghosh KN, Bhattacharya A, Adhya S. Leishmania DNA in Phlebotomus and Sergentomyia species during a kala-azar epidemic. Am J Trop Med Hyg. 1997;57:423-5.

34. Senghor M, Niang A, Depaquit J, Faye M, Ferté H, Faye B, et al. Canine leishmaniasis caused by Leishmania infantum transmitted by Sergentomyia species (Diptera: Psychodidae) in Senegal: ecological, parasitological and molecular evidences. In Proceedings of the 7th Internacional Symposium of Phlebotomine Sanflies: 25-30 April 2011; Izmir.

35. Kanjanopas K, Siripattanapipong S, Ninsaeng U, Hitakarun A, Jitkaew S, Kaewtaphaya $\mathrm{P}$, et al. Sergentomyia (Neophlebotomus) gemmea, a potential vector of Leishmania siamensis in southern Thailand. BMC Infect Dis. 2013;13:333.

36. Seblova V, Sadlova J, Carpenter S, Volf P. Development of Leishmania parasites in Culicoides nubeculosus (Diptera: Ceratopogonidae) and implications for screening vector competence. J Med Entomol. 2012;49:967-70.

37. Seblova V, Sadlova J, Carpenter S, Volf P. Speculations on biting midges and other bloodsucking arthropods as alternative vectors of Leishmania. Parasit Vectors. 2014;7:222

38. Sadlova J, Dvorak V, Seblova V, Warburg A, Votypka J, Volf P. Sergentomyia schwetzi is not a competent vector for Leishmania donovani and other Leishmania species pathogenic to humans. Parasit Vectors. 2013;6:186.

39. Volf $P$, Myskova J. Sand flies and Leishmania: specific versus permissive vectors. Trends Parasitol. 2007;23:91-2. 